\title{
2D Materials Armors Showing Superior Impact Strength of Few Layers
}

\author{
Stefano Signetti, ${ }^{\dagger}$ Simone Taioli, ${ }^{*},+\Phi$ and Nicola M. Pugno ${ }^{*, \dagger, \delta, \|}$ \\ †Laboratory of Bio-Inspired and Graphene Nanomechanics, \\ Department of Civil, Environmental and Mechanical Engineering, University of Trento, \\ via Mesiano 77, I-38123 Trento, Italy. \\ $\ddagger$ European center for Theoretical Studies in Nuclear Physics and Related Areas, \\ Fondazione Bruno Kessler $\&$ Trento Institute for Fundamental Physics and Applications, \\ strada delle Tabarelle 286, I-38123 Villazzano (Trento), Italy \\ \Faculty of Mathematics and Physics, Charles University, 18000 Praha 8, Czech Republic \\ $\S$ School of Engineering and Materials Science, Queen Mary University of London, \\ Mile End Road, E1 4 NS London, UK \\ \|Ket-Lab, Edoardo Amaldi Foundation, Italian Space Agency, via del Politecnico snc, \\ I-00133 Roma, Italy \\ E-mail: taioli@ectstar.eu; nicola.pugno@unitn.it
}

\begin{abstract}
We study the ballistic properties of two dimensional (2D) materials upon the hypervelocity impacts of $\mathrm{C}_{60}$ fullerene molecules combining ab initio density functional tight binding and finite element simulations. The critical penetration energy of monolayer membranes is determined using graphene and the $2 \mathrm{D}$ allotrope of boron nitride as case studies. Furthermore, the energy absorption scaling laws with variable number of layers and interlayer spacing are investigated, for homogeneous or hybrid configurations
\end{abstract}


(alternated stacking of graphene and boron-nitride). At the nano-level a synergistic interaction between layers emerges, not observed at the micro- and macro-scale for graphene armors. This size-scale transition in the impact behavior towards higher dimensional scales is rationalized in terms of scaling of the damaged volume and of material strength. An optimal number of layers, between 5 to 10, emerges demonstrating that few layers 2D materials armors possess impact strength even higher than their monolayer counterparts. These results provide fundamental understanding for the design of ultra-lightweight multilayer armors using enhanced 2D-materials-based nanocomposites.
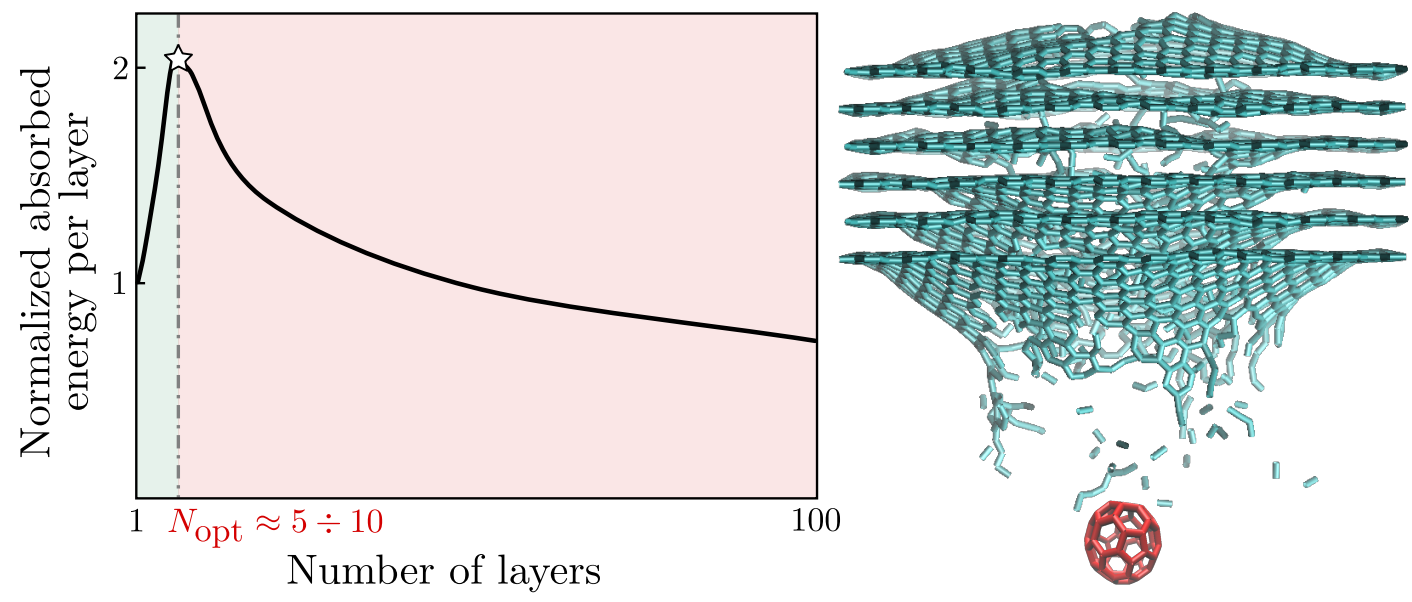

\section{Keywords}

Graphene, hexagonal boron-nitride, hypervelocity impact, nanoarmors, multiscale modeling

\section{Introduction}

The protection of structures and devices from the penetration of high-energy impacting projectiles is still an open issue for theoretical modeling and applied research, as well as relevant in several areas of technology, such as materials science and engineering, automotive, 
aerospace, and defense. For example, spacecrafts are commonly exposed during their operation to hypervelocity collisions (velocities $\geq 7-8 \mathrm{~km} / \mathrm{s}$ ) of micrometeoroids or orbital debris, ${ }^{1}$ leading to surface degradation, on-board instrumentation failures, up to complete perforation and structural damage. Other applications where impact assessment shows great deal of interest are in the field of stretchable and wearable electronics, ${ }^{2}$ where devices may undergo several and severe accidental shocks during their service life. Protection with a massive shield is straightforward but is often impracticable since lightness, flexibility, or ergonomics are of paramount importance in all these applications. Thus a growing interest towards the development of unconventional nanocomposites having high specific toughness and low weight has been witnessed. Solutions that embed 2D nanomaterials layers ${ }^{3,4}$ exploiting size-scale effects on mechanical properties are ideal candidates for such applications, increasing the resistance to shock loads while keeping the required flexibility. Furthermore, the possibility to exploit properties of embedded nano-material beyond the mere structural function ${ }^{5}$ can lead to further lightening of the system.

Among intercalated materials, graphene, along with extraordinary thermal, optical, ${ }^{6,7}$ and electrical properties,${ }^{8,9}$ shows outstanding fracture strength $(\sigma \approx 130 \mathrm{GPa})$ and Young's modulus $(E \approx 1 \mathrm{TPa})^{10}$ coupled with relatively low density $\left(\rho \approx 2200 \mathrm{~kg} / \mathrm{m}^{3}\right)$. According to the dimensional analysis carried out by Cuniff, ${ }^{11,12}$ the limiting penetration velocity of an homogeneous elastic barrier scales as $U^{1 / 3}$ where $U=\frac{\sigma \varepsilon}{2 \rho} \sqrt{\frac{E}{\rho}}$ is the product of the material specific dissipated energy times the wave speed in the considered medium, and $\varepsilon$ is the ultimate strain of the material. In this regard, graphene embedded into composite materials is an ideal candidate for impact protection, reaching unprecedented values of $U \approx 0.8 \cdot 10^{11} \mathrm{~m}^{3} / \mathrm{s}^{3}$ $(\varepsilon=\sigma / E=0.13)$. Indeed, it has been reported that graphene intercalation in conventional composite materials effectively increases their ballistic resistance. ${ }^{13}$ Other $2 \mathrm{D}$ materials such as the hexagonal allotrope of boron-nitride $(\mathrm{h}-\mathrm{BN})^{14}$ or molybdenum disulfide $\left(\mathrm{MOS}_{2}\right)^{15}$ also display excellent tensile properties and are equally promising. However, their tensile characteristics are lower than graphene and studies on these materials have been discarded 
due to the overwhelming interest in graphene-based structures.

While computational modeling of defect-free structures moght overestimate the mechanical properties of actual 2D-materials, however these latter ones usually outperform those of materials traditionally employed as energy absorbers, being able to guarantee the same level of protection against penetrating masses at $\approx 1 / 100$ in weight. Indeed, the remarkable mechanical properties of 2D materials have been confirmed using analytical methods based on continuum theories ${ }^{16,17}$ and computational atomistic models ${ }^{18}$ also in the presence of defects ${ }^{19}$ and in out-of-equilibrium configurations. ${ }^{20}$ In particular, layered graphene has been the subject of intensive experimental ${ }^{21,22}$ and computational ${ }^{21,23,24}$ investigations to evaluate its performance as ballistic material, showing great potential for its use in these applications. However, discrepancies in energy absorption capabilities between the atomistic scale and the micro-scale were reported, suggesting the presence of scaling effects.

Nevertheless, the search for unconventional materials with outstanding mechanical properties should be pursued hand-in-hand with the structural optimization ${ }^{3}$ in order to achieve specific mechanical requirements in a cost-effective and efficient way. In this regard, some studies investigated the role of spaced armors, also at the nanoscale. ${ }^{25}$ In a previous work ${ }^{26}$ we demonstrated how the monolithic solution for a composite laminate aimed at ballistic applications is tougher than the corresponding spaced counterpart due to synergistic interaction between layers. Furthermore, we identified optimal interface strength parameters for maximizing the specific energy absorption of the layers. This behavior suggests that the material structural arrangement, along with impact conditions, highly affects the impact properties, thus it is worth to be more thoroughly investigated.

From a methodological point of view, approaches beyond molecular dynamics (MD) based on classical force fields have not yet been widely used for studying the impact properties of 2D nanomaterials due to their high computational cost with increasing size of the system. Notable exceptions have been reported in modeling analogous problems, in which intermediate kinetic energy regimes (around tens of eV) were used to achieve the epitaxial growth of 
silicon carbide ${ }^{27-29}$ and graphene ${ }^{30,31}$ via buckyball beams impacting on silicon or metallic substrates.

In this work we investigate the ballistic behavior of 2D-materials-based armors, undergoing the hypervelocity impact of fullerene $\left(\mathrm{C}_{60}\right)$ using a multiscale approach, ranging from density functional tight-binding (DFTB) simulations at nanoscale, to finite element method (FEM) and continuum models at microscale. Graphene, h-BN and hybrid nanomaterials based on the alternate stacking of these 2D materials are taken as case studies. First, we determine the critical perforation conditions, and thus the intrinsic impact strength of these 2D-materials, by simulating ballistic curves of graphene and h-BN monolayers. Multilayer armor configurations, including heterogeneous mixing of layered materials (alternate stacking of graphene and h-BN), are then analyzed to understand the scaling of energy absorption capabilities. The latter investigations are aimed at understanding the modifications introduced in materials by using 2D structures as reinforcement in nanocomposites. Finally, ab initio DFTB simulations are supported and extended across dimensional scales by FEM and continuum models and compared with the experimental data available in the literature. ${ }^{21,22}$

\section{Methods}

DFTB atomistic model. First-principles simulations of fullerene-surface collisions were carried out within the framework of the DFTB approach. In this method, a second-order expansion of the full DFT electronic density is performed, resulting in an expression of the total energy of the system as a sum of three different contributions: ${ }^{32}$ tight-binding-like matrix elements, a Coulomb interaction, and a repulsive pair-potential. Usually, the terms appearing in the total energy expression are parametrised to reproduce accurately high-level electronic structure calculations for several different bonding conditions. In this way, the transferability of these pre-calculated terms (the so-called Slater-Koster matrix elements) to 
different chemical environments and physical conditions as well as a considerable reduction (around 2 orders of magnitude) of the computational cost of this approach with respect to full DFT simulations ${ }^{29,33}$ are generally obtained. Due to this substantial speed gain, DFTB can be used to simulate systems larger than those accessible by full DFT, to follow their dynamics for longer time scales and to test how the tuning of the DFTB parameters affects the impact dynamics at an affordable computational cost.

The computational supercell used in the impact calculations is tetragonal (see Figure S2 in the Supporting Information) and, after optimization of both atomic positions and lattice vectors, measures $48.83 \AA$ along the $x$-direction and $41.67 \AA$ along the $y$-direction for h-BN substrates, $47.96 \AA$ and $50.41 \AA$ for multi-layer graphene, and $50.08 \AA$ and $50.08 \AA$ for intercalated h-BN graphite multilayer, respectively. These dimensions were selected to have a ratio between the target supercell dimension $L$ and the fullerene mean nucleus to nucleus diameter $(d=7.06 \AA)$ greater than 6 . This threshold ratio allows one to obtain negligible influence of edge effects on the impact properties. Fullerene was separately optimized and initially placed at $5 \AA$ distance on the top of the slab. The supercell size was then increased of $5 \AA$ along the collision direction (thus orthogonal to the membrane plane) to avoid spurious interaction among periodic images due to the long-range part of the Coulomb potential. The dimension along the z-direction increased according to the number of layers considered and to the initial kinetic energy. The Brillouin zone was sampled at the $\Gamma$-point only, due to the large number of atoms in the calculation supercells, always larger than 1000 up to 6000, depending on the number of layers.

DFTB calculations were performed equilibrating the system at room temperature ( $\mathrm{T}=300 \mathrm{~K})$. We used a room temperature Fermi smearing for the electronic density, within the selfconsistent charge framework (SCC-DFTB) that leads to an improved description of the Coulomb interaction between atomic partial charges. DFTB interactions have been empirically corrected for the van der Waals (vdW) forces among carbon and boron nitride planes, since SCC-DFTB does not include these effects. In particular, the pair-wise Lennard-Jones 
potential was included between each pair of atoms with the parameters taken from the Universal Force Field (UFF). ${ }^{34}$ Atomistic simulations were performed in the micro-canonical ensemble (NVE), setting the time step to 1 fs to enforce total energy conservation and each simulation lasted 2 ps. Finally, the perimeter edge of each layer in all simulations was kept fully clamped during the dynamic evolution to simulate a material bulk. Electronic band structure is parametrised by the semi-relativistic, self-consistent charge Slater-Koster interatomic matrix element sets matsci-0-3. ${ }^{35}$ The DFTB + code suite was used to perform the ab-initio simulations. ${ }^{36}$

FEM model. Continuum models based on finite element method were developed and used to complement first-principles simulations. Indeed a major goal of our computer investigation is to build and calibrate a computational tool based on continuum mechanics to investigate impact problems on nano-membranes at lower computational cost. The graphene and h-BN membranes were modelled with thin shell elements with graphene and h-BN layer having a nominal thickness of $3.415 \AA$ and $3.407 \AA$, which correspond to their inter-layer equilibrium distance. ${ }^{37}$ The fullerene spherical impactor was modelled as a rigid shell body, having an external radius of $5.15 \AA$. The dissipated energy by internal deformation of the fullerene, not considered in the model, was conventionally taken into account for the a posteriori computation of the absorbed energy according to the computations of $\mathrm{Xu}$ and co-workers. ${ }^{38,39}$

The nanomembranes were modelled with fully integrated shells (2x2 Gauss points) based on the Reissner-Mindlin kinematic assumption. Since the graphene is assumed to experience large strains at impact, the constitutive response of the material is assumed to be elastic and isotropic with a non-linear law of the type $\sigma=E \varepsilon+B \varepsilon^{240}$ where $\sigma$ is the symmetric second Piola-Kirchhoff stress, $\varepsilon$ is the uniaxial Lagrangian strain, $E$ is the linear elastic modulus, and $B$ is the third-order non-linear elastic modulus. The law parameters for both graphene and boron nitride are determined according to DFT computations available elsewhere. ${ }^{18,41}$ The densities are $\rho_{\mathrm{G}}=2.2 \mathrm{~g} / \mathrm{cm}^{3}$, and $\rho_{\mathrm{h}-\mathrm{BN}}=2.1 \mathrm{~g} / \mathrm{cm}^{310,41}$ for graphene and h-BN respectively. 
Material failure was treated via an erosion algorithm based on the Lagrangian uniaxial strain $\varepsilon$. When the failure condition is reached at one of the element integration point, the element is deleted from the simulation (elastic strain energy properly accounted in the computations) and thus fracture can nucleate and propagate.

The molecular vdW interactions between graphene and h-BN layers with the fullerene projectile were taken into account by a cohesive model on the basis of the work by Jiang et al., ${ }^{42}$ under the hypothesis that the layers have an infinite extension in the plane $x y$. Considering two layers, the homogenized cohesive energy per unit area, function of the distance $r$ between two pair nodes, is the sum of the contributions of the potential energy $\Pi(r)$ of the $n$ atomic pairs a-b (C-C, B-B, N-N, C-B, C-N, B-N):

$$
\Phi=\sum_{i=1}^{n} 2 \pi \psi_{i, \mathrm{a}} \psi_{i, \mathrm{~b}} \int_{0}^{\infty} \Pi_{i}(r) z d z
$$

where, in our case, $\Pi(r)$ is a Lennard-Jones 6-12 functional form. The $\psi$ are the homogenization parameters to spread the discrete interaction of $\Pi(r)$ over a continuum equivalent surface. In particular, $\psi=\Gamma /\left(3 \sqrt{3} l_{0}^{2}\right)$ has the meaning of number of atoms per unit area, being $l_{0}$ the equilibrium $\mathrm{C}-\mathrm{C}$ or $\mathrm{B}-\mathrm{N}$ bond lengths before deformation, $\Gamma=4$ for $\mathrm{C}$ atoms in the graphene lattice and $\Gamma=2$ for $\mathrm{B}$ and $\mathrm{N}$ in the h-BN lattice. The cohesive stress-layer separation law is then obtained from derivation of the cohesive energy with respect to the normal and shear interface displacement (see Section S2 in the Supporting Information for more details on the derivation of the cohesive model and its implementation). The cohesive law in the multilayer is dominated by the first 3 closest layers and the contribution of further layer can be neglected (see Figure S1 in the Supporting Information). Such homogenization neglects the effect of lattice, being actually rather small. ${ }^{37}$ 


\section{Results and discussion}

\subsection{Ballistic properties of the single layers}

To compare the response of different thin armors upon impact it is a customary in ballistic analysis to plot the projectile residual velocity $V_{\text {res }}$ against its initial impact value $V_{0}$. This representation, also known in the field as ballistic curve, easily enables to discriminate between the projectile bouncing (ricochet) and the penetration regimes, thus identifying the critical penetration energy of the target. ${ }^{4-45}$ The initial velocity $\left(V_{0}\right)$ of the fullerene center of mass $(\mathrm{COM})$ is imposed within the range $3-15 \mathrm{~km} / \mathrm{s}$ orthogonally to the substrate layers (normal impact condition). The projectile residual center of mass kinetic energy $\left(K_{\text {res }}\right)$ and velocity $\left(V_{\text {res }}\right)$ are intended respectively as the translational kinetic energy and velocity that the fullerene COM reaches asymptotically after the collision. In DFTB simulations COM kinetic energy is calculated as difference between the total energy of the fullerene and its internal energy (see Section S1 in the Supporting Information), the latter being associated to the molecule shape distortion. A value very close to $0 \mathrm{eV}$ of the COM kinetic energy represents the fullerene molecule embedded in the layer and "almost at rest". The resulting ballistic curves for the graphene and h-BN monolayers are reported in the left panel of Figure 1, while the corresponding numerical values of $V_{\text {res }}$ and $K_{\text {res }}$ can be found in Table 1 .

In order to rationalize the result in the perforation regime we introduce a model based on the conservation of energy. The initial impact kinetic energy $K_{0}$, associated to the center of mass, is dissipated by the membrane after the complete projectile penetration by failure of a material volume defined by the layer thickness and the damaged area. Referring to the fullerene COM kinetic energies:

$$
K_{0}-K_{\mathrm{res}}=\frac{1}{2} M V_{0}^{2}-\frac{1}{2} M V_{\mathrm{res}}^{2}=\eta \bar{\sigma} \pi R^{2} t .
$$

where $M$ is the fullerene projectile mass, $\bar{\sigma}$ is the impact strength of the 2D-membrane, $t$ is 

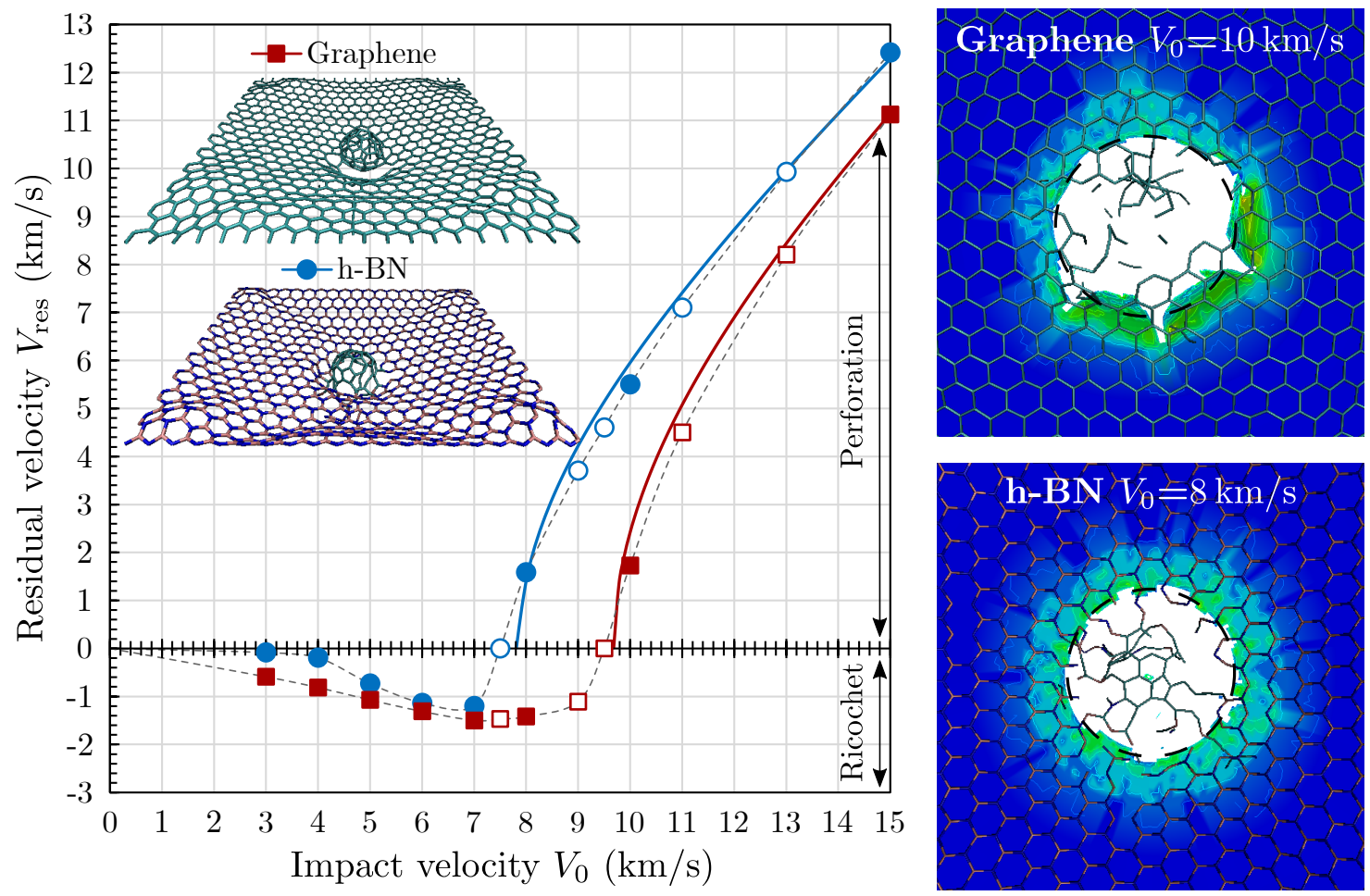

Figure 1: Left panel: ballistic curves of single layer graphene and h-BN, from DFTB (filled dots) and FEM (empty dots) simulations. The residual velocity $V_{\text {res }}$ is referred to the $\mathrm{C}_{60}$ center of mass (COM). Graphene provides a higher limit penetration velocity (and impact energy) than h-BN monolayer. Consequently, graphene provides lower residual velocity $V_{\text {res }}$ at perforation and a higher restitution coefficient in the ricochet regime. The dashed lines represent a guide to the eye while the continuous lines are derived from Equation (3) on the data corresponding to penetration regime. Right panel: configurations of graphene and h-BN at the penetration limit velocity with comparison between DFTB and FEM simulations. The contour plot of von-Mises stresses from FEM is also depicted. The equivalent damaged areas are highlighted and have a radius of $6.65 \AA$ for graphene and $6.39 \AA$ for boron nitride and are used for determining the material impact strength $\bar{\sigma}$. (See Videos S1S4 for impact DFTB simulations on h-BN at $8 \mathrm{~km} / \mathrm{s}$, on graphene at $10 \mathrm{~km} / \mathrm{s}$, and FEM simulations on graphene and h-BN at $13 \mathrm{~km} / \mathrm{s}$ ). 
Table 1: Residual kinetic energy $\left(K_{\text {res }}\right)$ and velocity $\left(V_{\text {res }}\right)$ obtained from DFTB and FEM impact simulations on single layer graphene and h-BN.

\begin{tabular}{|c|c|c|c|c|c|c|}
\hline \multirow[b]{2}{*}{$\begin{array}{c}K_{0} \\
{[\mathrm{eV}]}\end{array}$} & \multirow[b]{2}{*}{$\begin{array}{c}V_{\mathbf{0}} \\
{[\mathrm{km} / \mathrm{s}]}\end{array}$} & \multicolumn{2}{|c|}{ Graphene } & \multicolumn{2}{|c|}{ h-BN } & \multirow[b]{2}{*}{ Method } \\
\hline & & $\begin{array}{l}K_{\text {res }} \\
{[\mathrm{eV}]}\end{array}$ & $\begin{array}{c}V_{\text {res }} \\
{[\mathrm{km} / \mathrm{s}]}\end{array}$ & $\begin{array}{l}K_{\text {res }} \\
{[\mathrm{eV}]}\end{array}$ & $\begin{array}{c}V_{\text {res }} \\
{[\mathrm{km} / \mathrm{s}]}\end{array}$ & \\
\hline 33.63 & 3.0 & -1.30 & -0.59 & -0.03 & -0.09 & DFTB \\
\hline 59.78 & 4.0 & -2.47 & -0.81 & -0.14 & -0.19 & DFTB \\
\hline 93.41 & 5.0 & -4.30 & -1.07 & -2.00 & -0.73 & DFTB \\
\hline 134.51 & 6.0 & -6.43 & -1.31 & -4.83 & -1.14 & DFTB \\
\hline 183.09 & 7.0 & -8.41 & -1.50 & -5.37 & -1.20 & DFTB \\
\hline 209.88 & 7.5 & -8.07 & -1.47 & 0.00 & 0.00 & FEM \\
\hline 239.13 & 8.0 & -7.52 & -1.42 & 9.42 & 1.59 & DFTB \\
\hline 302.65 & 9.0 & -4.60 & -1.11 & 51.15 & 3.70 & FEM \\
\hline 336.73 & 9.5 & 0.00 & 0.00 & 79.06 & 4.60 & FEM \\
\hline 373.64 & 10.0 & 11.15 & 1.73 & 113.85 & 5.52 & DFTB \\
\hline 451.47 & 11.0 & 75.33 & 4.49 & 187.82 & 7.09 & FEM \\
\hline 630.56 & 13.0 & 253.08 & 8.23 & 364.73 & 9.88 & FEM \\
\hline 839.50 & 15.0 & 462.86 & 11.13 & 575.44 & 12.41 & DFTB \\
\hline
\end{tabular}

the thickness of the single layer, and $\eta$ is a damage parameter, whose physical meaning is the ratio between the effective damaged area of the perforated membrane versus the fullerene projected area $\pi R^{2}(R=5.15 \AA$ is the fullerene outer radius in the undeformed configuration, given by the sum of the half nucleus-to-nucleus fullerene diameter of $7.06 \AA$ and the mean carbon vdW radius of $1.62 \AA^{46}$ ). In order to include energy dissipation beyond the model considered here, the Equation (2) can be generalized as: ${ }^{47}$

$$
V_{\mathrm{res}}=\gamma\left(V_{0}^{p}-\frac{p\left(\eta \bar{\sigma} \pi R^{2} t\right)}{M}\right)^{\frac{1}{p}}
$$

where $p$ is theoretically equal to 2 for rigid projectile, and $\gamma$ is a model-dependent coefficient, which is equal to unity assuming that dissipation is due only to target deformation and thus projectile damage is not taken into account, as in Equation (2). From the best-fit of simulation data (Figure 2) corresponding to the penetration regime we find $\gamma \approx 0.975$, 0.958 and $p \approx 2.003,2.005$ for graphene and h-BN respectively. The impact strength can be 
estimated from the intercept of the linear fit of the $K_{\text {res }}-K_{0}$ curve (see Figure 2 ). In order to get a precise estimate of $\eta$ the actual damaged area was computed by measuring the mass of the eroded elements in the FEM simulations: we find $\eta_{\mathrm{G}}=3.61$ and $\eta_{\mathrm{h}-\mathrm{BN}}=3.33$ for graphene and h-BN respectively. Assuming an equivalent circular damaged area the corresponding radii are $R_{\mathrm{G}}=6.65 \AA$ and $R_{\mathrm{h}-\mathrm{BN}}=6.39 \AA$. Note that the damaged area increases, not monotonically, with the projectile impact energy (see Figure S3 in the Supporting Information) and the previous estimation refers to the critical penetration condition, corresponding to the measure of the intercept. In this way, we derive an impact strength $\bar{\sigma}_{\mathrm{G}} \approx 125 \mathrm{GPa}$ for graphene and $\bar{\sigma}_{\mathrm{h}-\mathrm{BN}} \approx 91 \mathrm{GPa}$. The estimated values are comparable with the tensile strength of the two materials, namely $130 \mathrm{GPa}$ for graphene ${ }^{10}$ and $108 \mathrm{GPa}$ for h-BN. ${ }^{48}$

These results show that graphene is tougher than h-BN, being higher the minimum (critical) initial energy $K_{\mathrm{c}}$ necessary to the fullerene molecule to penetrate the layer $\left(K_{\mathrm{c}, \mathrm{G}}=352 \mathrm{eV}\right.$ for graphene, corresponding to a critical velocity of about $V_{\mathrm{c}, \mathrm{G}}=9.7 \mathrm{~km} / \mathrm{s}$ while $K_{\mathrm{c}, \mathrm{h}-\mathrm{BN}}=227 \mathrm{eV}$ and $V_{\mathrm{c}, \mathrm{h}-\mathrm{BN}}=7.8 \mathrm{~km} / \mathrm{s}$ for h-BN). Figure 1 shows also the comparison between the two different membranes superimposing the top view of DFTB and FEM simulations at the two minimum velocities leading to complete perforation, that is $10 \mathrm{~km} / \mathrm{s}$ for graphene and $8 \mathrm{~km} / \mathrm{s}$ for h-BN. The comparison between the radius of the impact crater shows good agreement between the two approaches. Moreover, FEM simulations show how the stresses (von-Mises depicted in the figure) are highly localized around the hole within a distance from the impact point lower than three times the molecule radius $R$. Referring to the estimated damaged volume, the specific critical energies for the perforation of the monolayers are equal to $\bar{K}_{\mathrm{c}, \mathrm{G}}=51.8 \mathrm{MJ} / \mathrm{kg}$ and $\bar{K}_{\mathrm{c}, \mathrm{h}-\mathrm{BN}}=45.0 \mathrm{MJ} / \mathrm{kg}$ respectively.

\section{$3.2 \quad$ Ricochet regime}

If the impact kinetic energy $K_{0}$ is not sufficiently high to perforate the membranes (ricochet regime), the target will dissipate the kinetic energy by undergoing two different deformation mechanisms, that is bending or stretching of the membrane, in relation to its bending and 


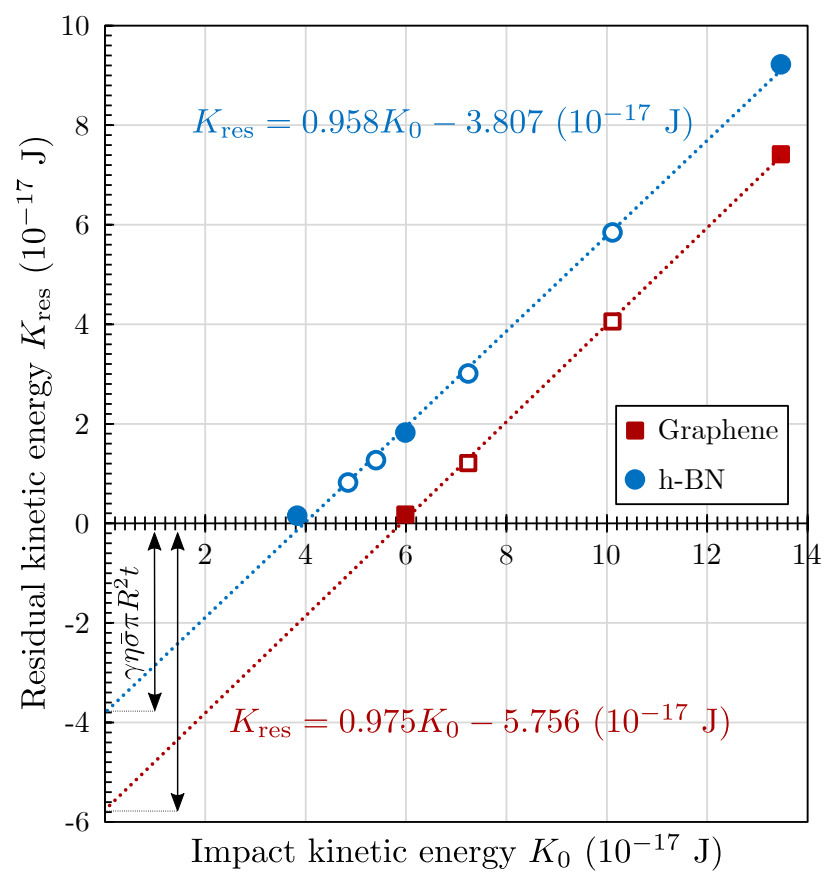

Figure 2: Plot of the fullerene residual COM energy $K_{\text {res }}$ vs. the initial impact energy $K_{0}$ at penetration regime (filled dots correspond to DFTB simulations, empty dots to FEM simulations). Assuming an energy dissipation within a material volume defined by the layer thickness $t$ and the projectile effective imprint area, nominally corresponding to the fullerene radius, the membrane impact strength is derived from the intercept of the linear fit (Equation (3), $p=2$ ). The damage parameter $\eta$ is the the ratio between the actual damaged area and the projected fullerene area. 
membrane stiffness, boundary conditions, and the impact kinetic energy of the projectile itself. We consider a configuration at which the instantaneous COM velocity of the projectile is close to zero, i.e. at the bouncing onset. Part of the energy is converted to vibration (phonons) of the membrane (bending or stretching); thus the elastic strain energy of target would be $K_{\text {strain }}=(1-f) K_{0}$ where $f$ represents the amount of the projectile kinetic energy dissipated by mechanical waves in the membrane plus the one converted into kinetic energy of the target and other forms of dissipation. To analyze the sub-critical regime, the target is assumed to be made of linear elastic and isotropic material, defined by the Young's modulus $E$ and Poisson's ratio $v$. Although the material properties are non-linear at high strain -as used in the FEM models- this simplification is acceptable far from the perforation and failure conditions. We model the system as a circular membrane of radius $L \gg R$ (as for the simulations) and thickness $t$. The circular membrane approximation is in good agreement with the armors' deformation, which is not affected by our choice of rectangular boundary scheme (see Videos S5-S6). Furthermore, this approximation implies a closed form solution. The impact is accounted as a normal concentrated force $F$ acting on the target. This force represents the counterpart only of the elastic strain energy $K_{\text {strain }}$. Considering a finite kinematic mechanism in a membrane regime, the vertical elastic displacement $w$ at the impact point satisfies: ${ }^{49}$

$$
\left(\frac{w}{t}\right)^{3}=\left[1-\left(\frac{1-3 \nu}{4}\right)^{1 / 3}\right]^{3} \frac{4 L^{2}}{(1+\nu) \pi E t^{4}} F .
$$

It follows that the relation between the membrane strain energy and the midspan vertical displacement $w$ under pure stretching regime is non-linear and, for the instant at which the projectile residual velocity is approximately close to zero (whereby projectile kinetic energy $K_{0}$ is almost completely transferred to the target), one can write $K_{\text {stretch }} \propto w^{4}$ :

$$
\begin{aligned}
K_{\text {stretch }} & =\int_{0}^{w} F(w) \mathrm{d} w \\
& =\left[1-\left(\frac{1-3 \nu}{4}\right)^{1 / 3}\right]^{-3} \frac{(1+\nu) \pi E t}{16 L^{2}} w^{4}
\end{aligned}
$$


Note that this result is analogous to the case of a cable subjected to a transversal concentrated force. On the other hand, if bending mechanism prevails the elastic strain energy for a clamped circular plate loaded at the center is $K_{\text {bend }}=1 / 2 k w^{2}$, with $k=16 \pi D / L^{2}$, $D=E t^{3} /\left[12\left(1-\nu^{2}\right)\right]$. It follows that, under bending, $K_{\text {bend }} \propto w^{2}$ :

$$
K_{\text {bend }}=\frac{2}{3} \pi \frac{E t^{3}}{\left(1-\nu^{2}\right)}\left(\frac{w}{L}\right)^{2} .
$$

To not overestimate the bending stiffness we used a reduced thickness of $t_{\mathrm{r}}=0.63 \AA=t / 5$ to match the bending properties of single-layer graphene. ${ }^{50}$ The elastic modulus is scaled accordingly $\left(E_{\mathrm{r}}\right)$ to maintain constant the tensile stiffness of the membrane $\left(E t=E_{\mathrm{r}} t_{\mathrm{r}}\right)$. The transition between the two deformation mechanisms takes place around a normalized displacement $w^{*} / L$ that can be determined by equating the two previous expressions for the absorbed kinetic energy (Equations (5),(6)):

$$
\frac{w^{*}}{L}=\frac{1}{\lambda} \sqrt{\left[1-\left(\frac{1-3 \nu}{4}\right)^{1 / 3}\right]^{3} \frac{32}{3(1+\nu)^{2}(1-\nu)}},
$$

where $\lambda=L / t_{\mathrm{r}}$ is the plate slenderness. The impact kinetic energy $K^{*}$ corresponding to the transition can be finally determined introducing Equation (7) either into Equation (5) or (6):

$$
K^{*}=\left[1-\left(\frac{1-3 \nu}{4}\right)^{1 / 3}\right]^{3} \frac{64 \pi E_{\mathrm{r}} t_{\mathrm{r}}^{3}}{9(1+\nu)\left(1-\nu^{2}\right)^{2}} \frac{1}{\lambda^{2}} .
$$

For the initial calculation, it is assumed that all the projectile kinetic energy $K_{0}$ is converted into strain energy of the target (i.e., $f=0$ ). Critical values of the impact energy and membrane midspan deflection, $K^{*}$ and $w^{*}$ respectively, which depend on the membrane material properties and geometrical configurations, define the transition between the membrane and bending deformation regimes. In particular, for $K_{0}<K^{*}$, or $w<w^{*}$, bending prevails and thus $K_{0} \propto w^{2}$, while for for $K_{0}>K^{*}$ or $w>w^{*}$ the plate undergoes prevailing stretching with $K_{0} \propto w^{4}$. The estimated transition displacement for both monolayers is 
Table 2: Maximum deflection $w$ at membrane midspan for single layer graphene and h-BN at different initial impact energies $K_{0}$ in the ricochet regime. At $K_{0}=239.1 \mathrm{eV}$ h-BN starts to show damage an thus it is not included in the computations.

\begin{tabular}{ccc}
\hline $\begin{array}{c}\text { Impact energy } \\
{[\mathbf{e V}]}\end{array}$ & $\begin{array}{c}w_{\mathbf{G}} \\
{[\mathbf{n m}]}\end{array}$ & $\begin{array}{c}w_{\mathbf{h}-\mathrm{BN}} \\
{[\mathbf{n m}]}\end{array}$ \\
\hline 33.6 & 0.370 & 0.456 \\
59.8 & 0.437 & 0.543 \\
93.4 & 0.493 & 0.619 \\
134.5 & 0.560 & 0.716 \\
183.1 & 0.628 & 0.781 \\
239.1 & 0.695 & perforated \\
\hline
\end{tabular}

$w^{*} / L \approx 0.03$ and it is independent of the material elastic modulus. Table 2 reports the recorded midspan deflection $w$ at different impact energies for the plates in the ricochet regime. The bi-logarithmic plot of Figure 3 shows the deflection $w$ as a function of the impact kinetic energy $K_{0}$. The estimated scaling exponents of the law $w=K_{0}^{s}$ are $s \approx 0.320$ for graphene and $s \approx 0.322$ for h-BN, and are intermediate between the predictions for stretching $(s=0.25)$ and bending $(s=0.5)$. From the best-fit of the simulation points (Table 2$)$ by using Equation (5) with $s=0.25$ we derive the elastic moduli of the materials, which are $\bar{E}_{\mathrm{G}} \approx 2.098 \mathrm{TPa}$ and $\bar{E}_{\mathrm{h}-\mathrm{BN}} \approx 0.815 \mathrm{TPa}$. According to the ratio between these theoretical predictions (computed assuming $f=0$ ) and the actual values of the mechanical properties ${ }^{10,14}$ we estimate $f_{\mathrm{G}} \approx 0.52$ and $f_{\mathrm{h}-\mathrm{BN}} \approx 0.12$ for graphene and h-BN membranes respectively. These values represent an estimate of the amount of projectile kinetic energy dissipated by mechanical waves. The corresponding transition kinetic energies, which are dependent on the respective elastic moduli, are $K_{\mathrm{G}}^{*} \approx 24 \mathrm{meV}$ and $K_{\mathrm{h}-\mathrm{BN}}^{*} \approx 20 \mathrm{meV}$, confirming that for the whole analyzed cases the plates mainly undergo stretching under impact.

\subsection{Energy scaling and optimal number of layers}

It is of paramount importance in multilayer armor design to know how the energy absorption capability scales with the addition of materials to test whether the material coupling is either 


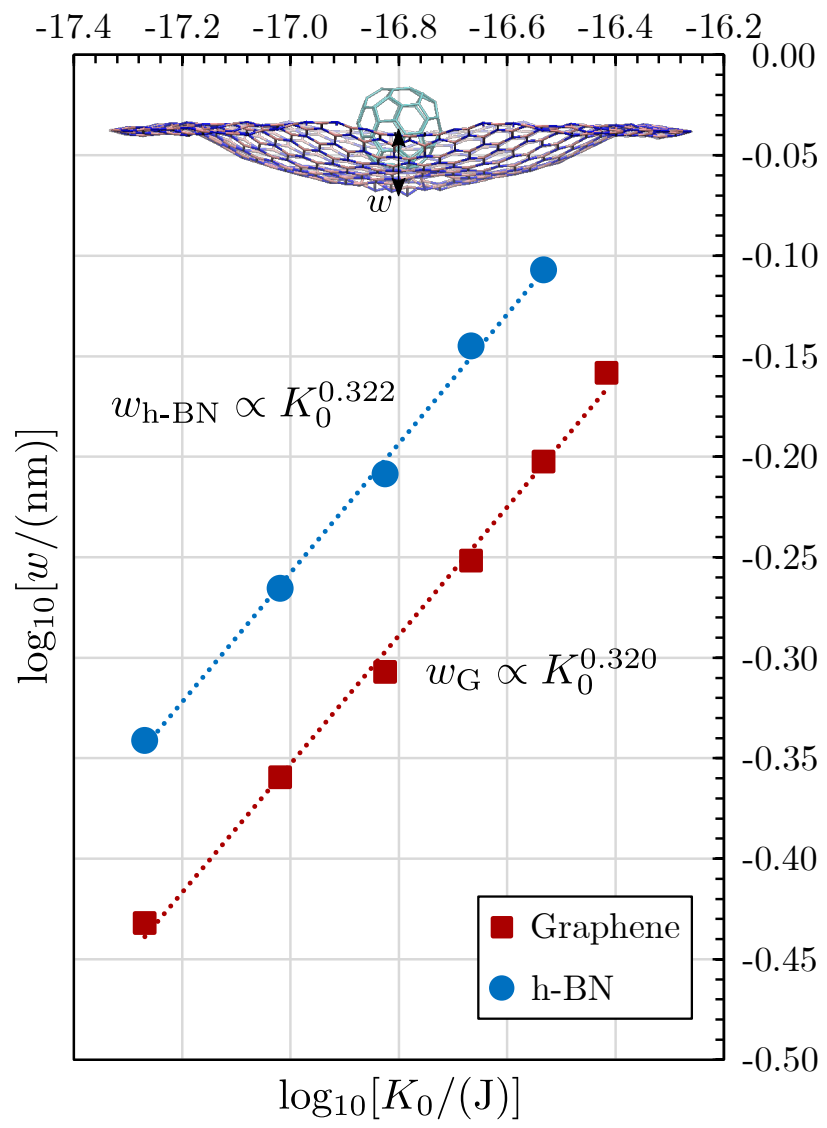

Figure 3: Plot of the maximum deflection $w$ at midspan vs. the impact kinetic energy $K_{0}$ of the incident particle for graphene and h-BN membranes in the ricochet regime. The obtained scaling $w \propto K_{0}^{0.32}$ is in proximity of the condition derived for the stretching regime $w \propto K_{0}^{0.25}$ and intermediate with that of bending $w \propto K_{0}^{0.5}$. 
efficient or not in the configurations of interest. Indeed, it has been experimentally observed in composite armors (and recently explained by continuum models ${ }^{26}$ ) that increasing the number of layers $n$ does not always results in an increase of the absorbed specific energy per layer. Depending on interface characteristics (e.g. adhesive strength), the layer coupling in some cases may be not effective, so that layers do not display synergistic behavior. We can express this concept by the following equation: ${ }^{26}$

$$
\frac{K_{\mathrm{abs}}(N)}{N}=K_{1} \cdot N^{\alpha}
$$

where $K_{1}$ is a constant. A scaling exponent $\alpha>0$ indicates a synergistic behavior in which single layers interact to mutually enhance their specific contribution. On the other hand, for $\alpha=0$, the total absorbed energy is the mere sum of single-layer contributions, while for $\alpha<0$ a sub-optimal behavior is identified in which increasing the number of layers leads to worse or inefficient inter-layer coupling. This inter-layer coupling results from the magnitude of the vdW interactions, i.e. ultimately the interface properties, and from the additional restrain that arises when the number of layer increases, changing from a thin- to thick- plate/bulk behavior. These factors will affect both the stress distribution within the target and its deformation capability, resulting at last in different protective capacity and in the possible scaling of energy absorption. DFTB simulations have been performed on 1, 2, 4, and 6 layer homogeneous and hybrid membranes with alternate stacking of graphene and h-BN. The used COM initial impact velocities were equal to 10, 15, 25, and $35 \mathrm{~km} / \mathrm{s}$ respectively, being slightly higher than the ballistic limit of the multilayers. The analyzed graphene-based, h-BN and hybrid nano-armors show all high positive values of $\alpha$ (Figure 4). From best fit we derive $K_{1, \mathrm{G}}=15.0 \mathrm{eV}$ and $K_{1, \mathrm{~h}-\mathrm{BN}}=14.5 \mathrm{eV}$ for the studied nanoscale impact configurations. However, this synergistic interaction between layers was not observed at the micro- and macro-scale for graphene armors, e.g. in the recent experimental work by Lee et al. ${ }^{21}$ on micrometric projectile impacts on graphite, in which a sub-optimal scaling law $(\alpha<0)$ was found. 

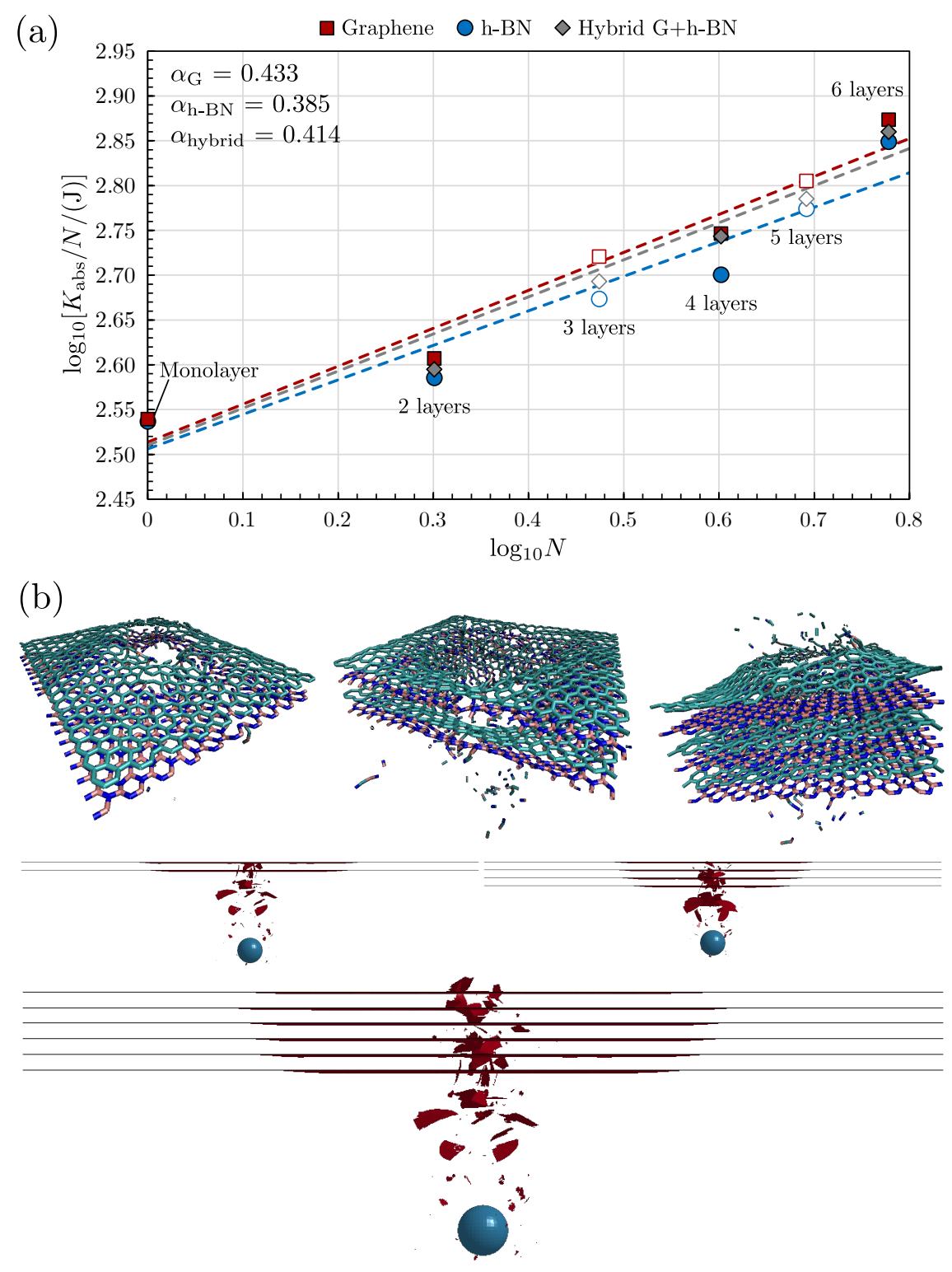

Figure 4: (a) Determination of the specific energy absorption scaling exponent $\alpha$ for graphene (red line), h-BN (blue line) and hybrid graphene/h-BN (grey line) alternate armors. Computed values of $\alpha$ are greater than 0 , showing a synergistic interaction as the number of layers increases. The fit for the determination of the scaling exponent is performed by using DFTB simulations (filled dots) while FEM simulation points (empty) are included for comparison. This result is far from being trivial since values of $\alpha<0$ have been found in macroscopic composite armors $^{26}$ and graphene upon microscale impact. ${ }^{21}$ (b) Impact simulations of the hybrid armor system (2, 4 and 6 layers) from DFTB (upper panel) and FEM (bottom panel) simulations are depicted. 
To explain this apparent mismatch we analyzed the evolution of the damaged volume, which can be directly correlated to the amount of the energy absorbed. During the perforation of the target the radius of the damaged area is not constant but increases through the thickness, creating a tapered damaged volume of truncated conical shape (see Figure S4 in the Supporting Information). The variable size of the radius at the $i$-th layer can be expressed by the following relation:

$$
R_{i}=\sqrt{\eta} R+\sum_{i=1}^{N} t_{i} \tan \theta
$$

where $\theta$ is the inclination angle of the cone apothem and $t_{i}$ is the thickness of each layer, that is, in our case, $3.415 \AA$ or $3.407 \AA$ for graphene or h-BN, respectively. For a shear-dominated mechanism $\theta \rightarrow 45^{\circ} .{ }^{44}$ Assuming all the layers of the same material $\left(t_{i}=t\right)$, Equation (10) yields to an evolution of the specific damaged volume as follows:

$$
\frac{V_{\mathrm{dam}}}{N}=\frac{\pi t^{3}}{3}\left[3 \eta\left(\frac{R}{t}\right)^{2}+3 \sqrt{\eta} N\left(\frac{R}{t}\right) \tan \theta+N^{2} \tan ^{2} \theta\right] .
$$

For the graphene membrane we determined from DFTB simulations $\theta \approx 13.5^{\circ}$ (Supporting Information, Figure S4). The shape of the truncated cone depends on the dimensional ratio $R / t$ between the radius of the impacting mass and the target thickness. However, the damaged area does not indefinitely increase as stated by Equation (11) but tends to saturate leading into a cylindrical volume, hence $\frac{V_{\text {dam }}}{N} \propto N^{0}$ for $N \rightarrow \infty$. In order to take into account this, Equation (11) is considered valid up to $N<N^{*}=\operatorname{int}[6 R / t]$, where $6 R$ is acknowledged in ballistic literature to be a reasonable value of the maximum radius of the damaged cone (see Figure S2 in the Supporting Information for the determination of the models supercells). Thus, for $N>N^{*}$ a constant asymptotic value of $R_{\max }=R\left(N^{*}\right)$ is assumed. Furthermore, accounting for a scaling law of the material strength $\sigma,{ }^{51}$ one can assess the strength from 
the following relation:

$$
\sigma=\sigma_{0}\left[(N+1) \cdot\left(\frac{R}{t}\right)\right]^{-\beta}
$$

where $\beta$ is the strength scaling exponent, and $\sigma_{0}$ the ideal material strength. In particular, the characteristic dimension of the material defect is assumed proportional both to $N$, i.e. the plate thickness, and to the area affected by the impact, which is directly proportional to $R$. The combination of volume and strength size-scalings, which both depend on $N$ and $R / t$, may yield in some cases -according to the competition between the two- to an optimal configuration as reported in Figure $5(\mathrm{a}) . N_{\mathrm{opt}}$ is the number of layer that characterizes the transition between positive and negative scaling, and that maximizes the specific energy absorption of the plate by means of strain. The evolution of the energy absorbed by the plate can be obtained as follows from Equations (11),(12):

$$
\frac{K_{\mathrm{abs}}(N)}{N}=\frac{1}{N}\left\{f K_{\mathrm{dyn}}(N)+(1-f) \sigma_{0} V_{\mathrm{dam}}(N)\left[(N+1) \cdot\left(\frac{R}{t}\right)\right]^{-\beta}\right\}
$$

where we assume $\beta=0.5$ according to Linear Elastic Fracture Mechanics and $f$ is again the coefficient that accounts for the amount of dissipated energy via mechanical waves $\left(K_{\mathrm{dyn}}\right)$, here assumed equal to 0 and independent of $N$.

Figure 5(b) reports the specific energy absorption versus the number of layers $N$ for different values of $R / t$. It can be clearly seen that $N_{\text {opt }}$ emerges for nanoscale configurations $(R / t<2)$, such as the ones investigated in this work with the fullerene impact $(R / t \approx 1.48)$. Our DFTB-FEM simulation results are in good quantitative agreement with the analytical prediction at the nanoscale. The optimal number of layer $N_{\text {opt }}$ is predicted to be 5 for $R / t=1$ and 10 for $R / t=2$, and from the coupled DFTB-FEM data we obtain $N_{\text {opt }}=7$. Furthermore, values of the absorbed energy at the nanoscale are in good agreement also with molecular dynamics simulations of Haque et al. ${ }^{52}$ at a comparable scale. The difference in the critical penetration energy with the microscale values obtained by Lee et al., ${ }^{21}$ calculated there in the order of $1 \mathrm{MJ} / \mathrm{kg}$, can be attributed in principle to the size scaling of strength 
(see Equation (12)). Moreover, despite the specific impact kinetic energies over the impact area are similar, $K_{0} /\left(\pi R^{2} t\right) \approx$, the experimental velocities investigated by Lee et al. are below the hypervelocity regime. These velocities result in a different damage mechanism, governed in the latter work by circumferential and radial crack formation and propagation rather than diffused and localized damage of the impact zone as in our simulations. For higher scales $(R / t>2)$ the optimum value vanishes and the scaling is negative for any $N$. For $R / t<10$ the contribution to positive scaling of the damaged volume is still relatively significant and a change in the slope $\alpha$ of the curves in the bi-logaritmic plane still appears. By increasing the dimension of the projectile, the specific damaged volume tends to be constant (Equation (11)) and the scaling of the strength is predominant (Figure 5(b)), determining a nearly constant negative $\alpha$ independent of $N$. Despite the damage mechanisms between our nanoscale simulations and microscale experiments by Lee et al. ${ }^{21}$ are different, our model is able to predict a negative scaling at the microscale. Thus, this behavior deserves further experimental investigation by perfoming microscale experiments also at higher impact velocities.

We finally studied the role of the spacing of the layers on the absorption capabilities. DFTB simulations have been performed on 2 and 4 layer graphene armors increasing by steps of $0.5 \AA$ the distance between the layers up to 3 times the standard vdW distance $(3.4 \AA)$, which is practically identical in multi-layer graphene and h-BN despite presenting major differences in the nature of chemical bonds and static polarizabilities. ${ }^{37}$ Impacts on multilayer targets are set up at the minimal velocity necessary for perforation of all layers, i.e. $15 \mathrm{~km} / \mathrm{s}$ for 2 layer systems and $25 \mathrm{~km} / \mathrm{s}$ for the 4 layer systems. Figure 6 reports the specific absorbed energy $K_{\mathrm{abs}} / N$ as a function of the standard spacing multiplier (absorbed energy values are reported in Table 3). No significant effect has been found in the analyzed domain, with a maximum difference of $3 \%$ between the standard spacing and the $3 \mathrm{x}$ spacing $(10.05 \AA)$, with the standard spacing being the toughest solution. 

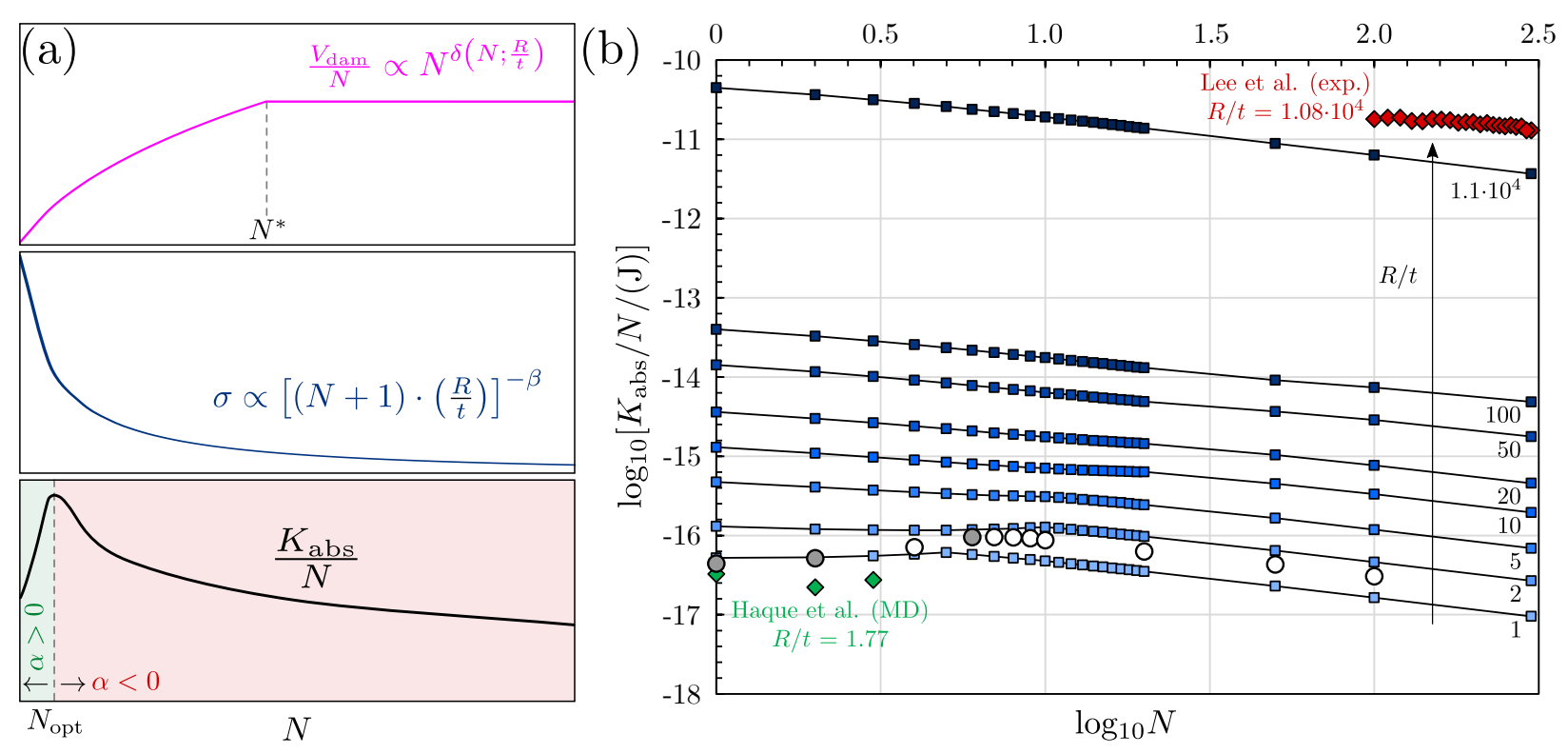

Figure 5: Scaling of the specific energy absorption in the multilayer nanoarmors with the dimensional scale. (a) Conceptual representation of the role of the damaged volume scaling (Equation (11)) and of the material strength scaling (Equation (12)) according to LEFM in determining an optimal number of layer $N_{\text {opt }}$, to which correspond both the maximum specific energy absorption by strain and the inversion in the sign of the scaling exponent $\alpha$. (b) Results obtained from analytical calculations (Equation (13)) for different $R / t$ configurations (curves with square dots, the lines are just a guide to the eye being the function of integers values of $N$ ) compared with the results from DFTB and FEM simulations (circular dots, filled and empty respectively), MD results from Haute et al. ${ }^{52}$ at the nanoscale, and experimental results from Lee et al. ${ }^{21}$ at the microscale.

Table 3: Absorbed energies for 2 and 4 layers graphene armors as a function of the layer spacing expressed as multiplier of the vdW distance.

\begin{tabular}{ccc}
\hline Layers spacing & $\begin{array}{c}K_{\text {abs }}[\mathrm{eV}] \\
\text { 2 layers }\end{array}$ & $\begin{array}{c}K_{\text {abs }}[\mathrm{eV}] \\
4 \text { layers }\end{array}$ \\
\hline $1.0 \mathrm{x}$ & 768 & 2333 \\
$1.5 \mathrm{x}$ & 766 & 2310 \\
$2.0 \mathrm{x}$ & 764 & 2290 \\
$2.5 \mathrm{x}$ & 762 & 2270 \\
$3.0 \mathrm{x}$ & 760 & 2250 \\
\hline
\end{tabular}



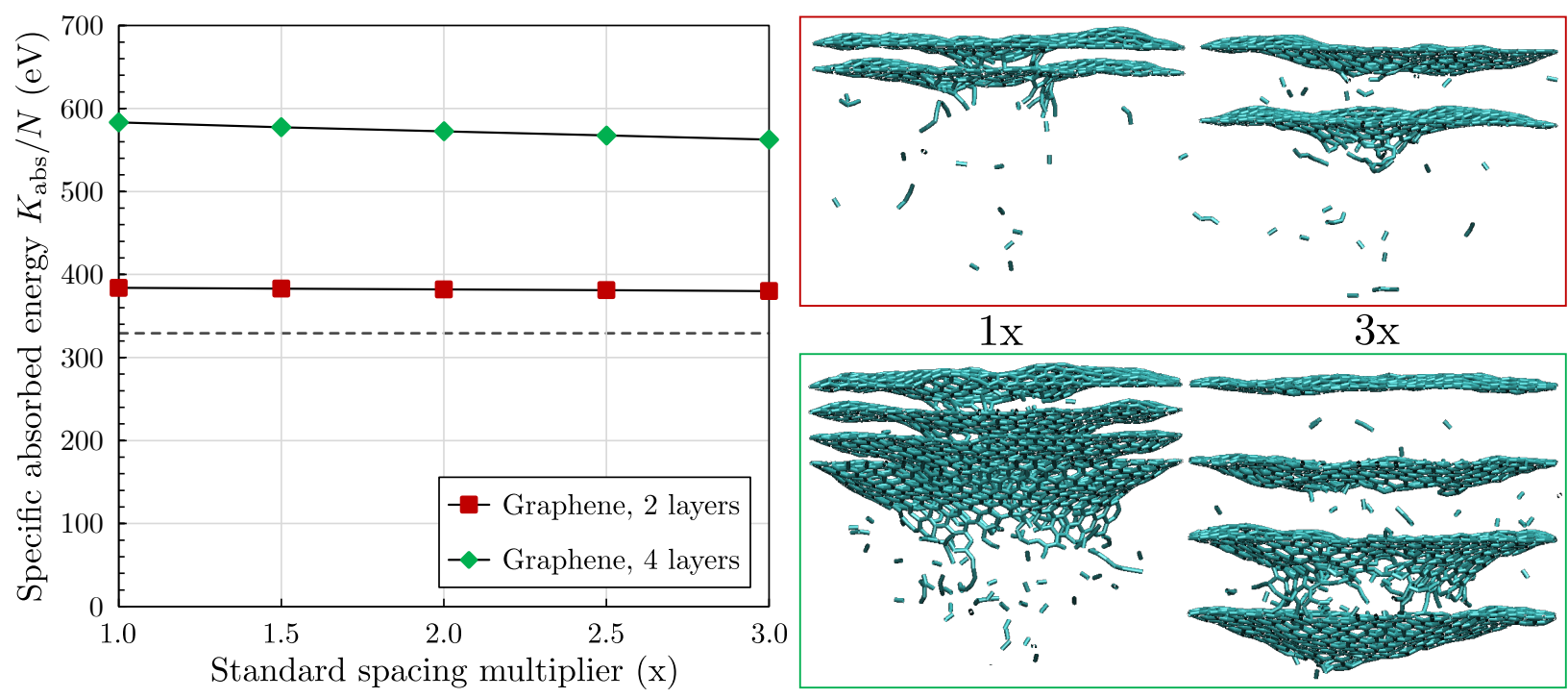

Figure 6: Left panel: specific energy absorption of 2 and 4 layer graphene with variable spacing. Comparison with the specific energy absorption of $330 \mathrm{eV}$ impact on single layer graphene confirms the synergistic interaction between layers at the nanoscale. Right panel: snapshots of the impact simulations on the reference armors with normal graphite spacing $(0.34 \mathrm{~nm})$ and three times this value are depicted.

\section{Conclusion}

In this work we studied the mechanical behavior of single and multilayer graphene and h-BN armors subjected to hypervelocity impacts of a $\mathrm{C}_{60}$ fullerene molecule. Coupling atomistic DFTB and continuum FEM approaches, the ballistic critical penetration energies of single sheets of graphene and h-BN were determined along with the impact strength of these 2D materials.

The membrane behavior in the subcritical impact regime (no perforation) was rationalized via a kinematic model on an elastic equivalent continuum membrane. The results found on homogeneous and hybrid multilayers suggest possible optimized designs at the nanoscale. The interlayer synergy could be increased by realizing a series of stacked pillared layers, a solution that has already been demonstrated doable in both computational ${ }^{20}$ and experimental ${ }^{53}$ studies. This solution would also realize a stable spaced configuration with interlayer distance higher than the vdW equilibrium, allowing to maximize the energy dissipation by membrane mechanism before the contact between adjacent layer occurs. This guideline is likewise 
applicable to both h-BN and hybrid armors since the critical displacement, with the exception of the Poisson's ratio, is independent of the membrane elastic properties.

The investigation on multilayer graphene structures has then been extended across different dimensional scales. We have demonstrated that generally at the microscale the scaling of these nanoarmors is not optimal, confirming that graphite is a weaker configuration also for impact loads. However moving to nanoscale -that is projectile dimension comparable with the thickness of the monolayer, $R / t \rightarrow 1$, and few layer armors, $N<10$ - a strong synergistic coupling emerges. This dimensional scaling is rationalized by taking into account both the damaged volume and the material strength scalings, according to the LEFM model. Our approach suggests a transition between positive and negative scaling at different dimensional scales which deserves further experimental investigation. At the nanoscale an optimal number of layers, between 5 to 10, emerges that maximizes also the specific energy dissipation under impact. These results suggest that multilayer 2D material based armors should be structured and optimized at the nanolevel, not relying on the mere high specific mechanical properties of the constituent materials. These armors, for example, would be particularly effective in providing protection of spacecrafts, especially deployable ones, and related instrumentation from high energy nanoscopic-size space dust ${ }^{54}$ or even suitable as coating for protection of ship propellers from erosion caused by fluid acoustic cavitation. ${ }^{55}$

\section{Supporting Information Available}

The Supporting Information is available free of charge on the ACS Publication Website at the DOI: http://dx.doi.org/10.1021/acsami.7b12030. 


\section{Authors information}

\section{Corresponding authors}

Simone Taioli - E-mail: taioli@ectstar.eu

Nicola Pugno - E-mail: nicola.pugno@unitn.it

\section{ORCID}

Stefano Signetti: 0000-0003-4128-0953

Simone Taioli: 0000-0003-4010-8000

Nicola Pugno: 0000-0003-2136-2396

\section{Present address}

SS is currently affiliated with the Department of Mechanical Engineering, Korea Advanced Institute of Science and Technology (KAIST), 291 Daehak-ro, Yuseong-gu, Daejeon, 34141 Republic of Korea.

\section{Notes}

The authors declare no competing financial interest.

\section{Acknowledgement}

ST is supported by the European commission under the Graphene Flagship (WP12 "Energy Storage", no. 696656). NMP is supported by the European Research Council (ERC PoC 2015 SILKENE, no. 693670) and by the European Commission H2020 under the Graphene Flagship (WP14 "Polymer Composites", no. 696656) and under the FET Proactive ("Neurofibres" no. 732344). This work used the ARCHER UK National Supercomputing Service (http: //www.archer.ac.uk) and the KORE computing cluster at FBK (https://sit. flok. 
eu/it/node/651). Access to computing and storage facilities owned by parties and projects contributing to the National Grid Infrastructure MetaCentrum, provided under the programme "Projects of Large Research, Development, and Innovations Infrastructures" (CESNET LM2015042), is also greatfully acknowledged (https://www. metacentrum. $\mathrm{cz} / \mathrm{en} /)$.

\section{References}

(1) NASA, International Space Station Risk of Impact from Orbital Debris. 2015; http: //www.nasa.gov/externalflash/iss_impact_risk/.

(2) Anagnostopoulos, G.; Pappas, P.-N.; Li, Z.; Kinloch, I. A.; Young, R. J.; Novoselov, K. S.; Lu, C. Y.; Pugno, N. M.; Parthenios, J.; Galiotis, C.; Papagelis, K. Mechanical Stability of Flexible Graphene-based Displays. ACS Appl. Mater. Interfaces 2016, 8, 2260522614 .

(3) Lee, J.-H.; Veysse, D.; Singer, J. P.; Retsch, M.; Saini, G.; Pezeril, T.; Nelson, K. A.; Thomas, E. L. High Strain Rate Deformation of Layered Nanocomposites. Nat. Commun. 2012, 3, 1-7.

(4) Androulidakis, C.; Koukara, E. N.; Frank, O.; Tsoukleri, G.; Sfyris, D.; Parthenios, J.; Pugno, N. M.; Papagelis, K.; Novoselov, K. S.; Galiotis, C. Failure Processes in Embedded Monolayer Graphene under Axial Compression. Sci. Rep. 2014, 4, 5271.

(5) Ramanathan, T.; Abdala, A. A.; Stankovich, S.; Dikin, D. A.; Herrera-Alonso, M.; Piner, R. D.; Adamson, D. H.; Schniepp, H. C.; Chen, X.; Ruoff, R. S.; Nguyen, S. T.; Aksay, I. A.; Prud'Homme, R. K.; Brinson, L. C. Functionalized Graphene Sheets for Polymer Nanocomposites. Nat. Nanotechnol. 2008, 3, 327-331.

(6) Umari, P.; Petrenko, O.; Taioli, S.; Souza, M. M. D. Electronic Band Gaps of Semicon- 
ducting Zig-zag Carbon Nanotubes from Many-body Perturbation Theory Calculations. J. Chem. Phys. 2012, 136, 181101.

(7) Taioli, S.; Umari, P.; Souza, M. M. D. Electronic Properties of Extended Graphene Nanomaterials from GW Calculations. Phys. Status Solidi B 2009, 246, 2572-2576.

(8) Kholmanov, I. N.; Magnuson, C. W.; Piner, R.; Kim, J. Y.; Aliev, A. E.; Tan, C.; Kim, T. Y.; Zakhidov, A. A.; Sberveglieri, G.; Baughman, R. H.; Ruoff, R. S. Optical, Electrical, and Electromechanical Properties of Hybrid Graphene-Carbon Nanotube Films. Adv. Mater. 2015, 27, 3053-3059.

(9) Chen, C.; Wu, J. Z.; Lam, K. T.; Hong, G.; Gong, M.; Zhang, B.; Lu, Y.; Antaris, A. L.; Diao, S.; Guo, J.; Dai, H. Graphene Nanoribbons Under Mechanical Strain. Adv. Mater. 2015, 27, 303-309.

(10) Lee, C.; Wei, X.; Kysar, J. W.; Hone, J. Measurement of the Elastic Properties and Intrinsic Strength of Monolayer Graphene. Science 2008, 321, 385-388.

(11) Cuniff, P. M. Dimensionless Parameters for Optimization of Textile-based Body Armor Systems. Proc. Int. Symp. Ballist., 18th. 1999; pp 1303-1310.

(12) Cuniff, P. M. Analysis of the System Effects in Woven Fabrics Under Ballistic Impact. Text. Res. J. 1992, 62, 495-509.

(13) Ávila, A. F.; Neto, A. S.; Jr., H. N. Hybrid Nanocomposites for Mid-range Ballistic Protection. Int. J. Impact Eng. 2011, 38, 669-676.

(14) A. Falin, a. Q. C.; Santos, E. J. G.; Scullion, D.; Qiang, D.; Zhang, R.; Yang, Z.; Huang, S.; Watanabe, K.; Taniguchi, T.; Barnett, M. R.; Chen, Y.; Ruoff, R. S.; Lee, L. H. Mechanical Properties of Atomically Thin Boron Nitride and the Role of Interlayer Interactions. Nat. Commun. 2017, 8, 15815. 
(15) Cooper, R. C.; Lee, C.; Marianetti, C. A.; Wei, X.; Hone, J.; Kysar, J. W. Nonlinear Elastic Behavior of Two-dimensional Molybdenum Disulfide. Phys. Rev. B 2013, 87, 035423.

(16) Pugno, N. M. A General Shape/Size-effect Law for Nanoindentation. Acta Mater. 2007, $55,1947-1953$.

(17) Pugno, N. M. Dynamic Quantized Fracture Mechanics. Int. J. Fract. 2006, 140, 159-168.

(18) Xu, M.; Paci, J. T.; Oswald, J.; Belytschko, T. A Constitutive Equation for Graphene Based on Density Functional Theory. Int. J. Solids Struct. 2012, 49, 2582-2589.

(19) Cranford, S. W. When is 6 Less than 5? Penta- to Hexa-Graphene Transition. Carbon 2016, 96, 421-428.

(20) Garberoglio, G.; Pugno, N. M.; Taioli, S. Gas Adsorption and Separation in Realistic and Idealized Frameworks of Organic Pillared Graphene: A Comparative Study. J. Phys. Chem. C 2016, 119, 1970-1978.

(21) Lee, J. H.; Loya, P. E.; Loeu, J.; Thomas, E. L. Dynamic Mechanical Behavior of Multilayer Graphene via Supersonic Projectile Penetration. Science 2014, 346, 10921096.

(22) M. J Eller, C. K. L.; Della-Negra, S.; Clubb, A. B.; Kim, H.; Young, A. E.; Schweikert, E. A. Hypervelocity Nanoparticle Impacts on Free-standing Graphene: A Sui Generis Mode of Sputtering. J. Chem. Phys. 2015, 142, 044308.

(23) Wetzel, E. D.; Balu, R.; Beaudet, T. D. A Theoretical Consideration of the Ballistic Response of Continuous Graphene Membranes. J. Mech. Phys. Solids 2015, 99, 23-31.

(24) Yoon, K.; Ostadhossein, A.; van Duin, A. D. T. Atomistic-scale Simulations of the Chemomechanical Behavior of Graphene under Nanoprojectile Impact. Carbon 2016, $99,58-64$. 
(25) Shang, H.; Wang, W. Hypervelocity Impact Properties of Graphene Armor via Molecular Dynamics Simulations. Proc. of the 10th Int. DYMAT Conf. Freiburg, Germany, 2012; p 04027.

(26) Signetti, S.; Pugno, N. M. Evidence of Optimal Interfaces in Bio-inspired CeramicComposite Panels for Superior Ballistic Protection. J. Eur. Ceram. Soc. 2014, 34, $2823-2831$.

(27) Verucchi, R.; Aversa, L.; Nardi, M. V.; Taioli, S.; a Beccara, S.; Alfè, D.; Nasi, L.; Rossi, F.; Salviati, G.; Iannotta, S. Epitaxy of Nanocrystalline Silicon Carbide on Si(111) at Room Temperature. J. Am. Chem. Soc. 2012, 134, 17400-17403.

(28) Taioli, S.; Garberoglio, G.; Simonucci, S.; a Beccara, S.; Aversa, L.; Nardi, M.; Verucchi, R.; Iannotta, S.; Dapor, M.; Alfè, D. Non-Adiabatic Ab Initio Molecular Dynamics of Supersonic Beam Epitaxy of Silicon Carbide at Room Temperature. J. Chem. Phys. 2013, 138, 044701.

(29) Aversa, L.; Taioli, S.; Nardi, M. V.; Tatti, R.; Verrucchi, R.; Iannotta, S. The Interaction of $\mathrm{C}_{60}$ on $\mathrm{Si}(111) 7 \times 7$ Studied by Supersonic Molecular Beams: Interplay between Precursor Kinetic Energy and Substrate Temperature in Surface Activated Processes. Front. Mater 2015, 2, 1-9.

(30) Tatti, R.; Aversa, L.; Verrucchi, R.; Cavaliere, E.; Garberoglio, G.; Pugno, N. M.; Speranza, G.; Taioli, S. Synthesis of Single Layer Graphene on $\mathrm{Cu}(111)$ by $\mathrm{C}_{60}$ Supersonic Molecular Beam Epitaxy. RSC Adv. 2016, 6, 37982-37993.

(31) Taioli, S. Computational Study of Graphene Growth on Copper by First-Principles and Kinetic Monte Carlo Calculations. J. Mol. Model. 2014, 20, 2260.

(32) Frauenheim, T.; Seifert, G.; Elstner, M.; Niehaus, T.; Köhler, C.; Amkreutz, M.; Sternberg, M.; Hajnal, Z.; Carlo, A. D.; Suhai, S. Atomistic Simulations of Complex 
Materials: Ground-state and Excited-state Properties. J. Phys.: Condens. Matter 2002, 14, 3015-3047.

(33) Garberoglio, G.; Taioli, S. Modeling Flexibility in Metal-Organic Frameworks: Comparison between Density-Functional Tight-Binding and Universal Force Field Approaches for Bonded Interactions. Microporous Mesoporous Mater. 2012, 163, 215-220.

(34) Rappe, A. K.; Casewit, C. J.; Colwell, K. S.; Goddard, W. A.; Skiff, W. M. UFF, a Full Periodic Table Force Field for Molecular Mechanics and Molecular Dynamics Simulations. J. Am. Chem. Soc. 1992, 114, 10024-10035.

(35) Frenzel, J.; Oliceira, A. F.; Jardillier, N.; Heine, T.; Seifert, G. Semi-relativistic, Self-consistent Charge Slater-Koster Tables for Density-Functional Based Tightbinding (DFTB) for Materials Science Simulations. 2009; http://www.dftb.org/ parameters/download/matsci/matsci-0-3/.

(36) Aradi, B.; Hourahine, B.; Frauenheim, T. DFTB+, a Sparse Matrix-based Implementation of the DFTB Method. J. Phys. Chem. A 2007, 11, 5678-5684.

(37) Hod, O. Graphite and Hexagonal Boron-Nitride have the Same Interlayer Distance. Why? J. Chem. Theory Comput. 2012, 8, 1360-1369.

(38) Xu, J.; Sun, Y.; Wang, B.; Li, Y.; Xiang, Y.; Chen, X. Molecular Dynamics Simulation of Impact Response of Buckyballs. Mech. Res. Commun. 2013, 40, 8-12.

(39) Xu, J.; Li, Y.; Xiang, Y.; Chen, X. A Super Energy Mitigation Nanostructure at High Impact Speed Based on Buckyball System. PLoS ONE 2013, 8, e64697.

(40) Pugno, N. M.; Marino, F.; Carpinteri, A. Towards a Periodic Table for the Nanomechanical Properties of the Elements. Int. J. Solids Struct. 2006, 43, 5647-5657.

(41) Boldrin, L.; Scarpa, F.; Chowdhury, R.; Adhikari, S. Effective Mechanical Properties of Hexagonal Boron Nitride Nanosheets. Nanotechnology 2011, 22, 505702. 
(42) Jiang, L. Y.; Huang, Y.; Jiang, H.; Ravichandran, G.; Gao, H.; Hwang, K. C.; Liu, B. A Cohesive Law for Carbon Nanotube/Polymer Interfaces Based on the van der Waals Force. J. Mech. Phys. Solids 2006, 54, $2436-2452$.

(43) Goldsmith, W. J. Impact - The theory and Physics of Colliding Solids, 2nd ed.; Dover Publications, 2001.

(44) Abrate, S. Impact on Composite Structures, 1st ed.; Cambridge University Press, 2001.

(45) Abrate, S. Ballistic Impact on Composites. Proc. Int. Conf. Compos. Mater., 16th. Kyoto, Japan, 2007; pp 1-10.

(46) Adams, G. B.; O'Keeffe, M.; Ruoff, R. S. Van Der Waals Surface Areas and Volumes of Fullerenes. J. Phys. Chem. 1994, 98, 9465-9469.

(47) Recht, R. F.; Ipson, T. W. Ballistic Perforation Dynamics. J. Appl. Mech. 1963, 30, 384-390.

(48) Wu, J.; Wang, B.; Wei, Y.; Yang, R.; Dresselhaus, M. Mechanics and Mechanically Tunable Band Gap in Single-Layer Hexagonal Boron-Nitride. Mater. Res. Lett. 2013, 1, 200-206.

(49) Chen, S. L.; Zheng, Z. L. Large Deformation of Circular Membrane under the Concentrated Force. Appl. Math. Mech. 2003, 24, 28-31.

(50) Scarpa, F.; Adhikari, S.; Gil, A. J.; Remillat, C. The Bending of Single Layer Graphene Sheets: the Lattice Versus Continuum Approach. Nanotechnology 2010, 21, 125702.

(51) Pugno, N. M. The Role of Defects in the Design of Space Elevator Cable: From Nanotube to Megatube. Acta Mater. 2007, 55, 5269-5279.

(52) Haque, B. Z. G.; Chowdhury, C. S. C.; Gillespie, J. W. Molecular Simulations of Stress Wave Propagation and Perforation of Graphene Sheets Under Transverse Impact. Carbon 2016, 102, 126-140. 
(53) Kumar, R.; Suresh, V. M.; Maji, T. K.; Rao, C. N. R. Porous Graphene Frameworks Pillared by Organic Linkers with Tunable Surface Area and Gas Storage Properties. Chem. Commun. 2014, 50, 2015-2017.

(54) Westphal, A. J. et al. Final Reports of the Stardust Interstellar Preliminary Examination. Meteorit. Planet. Sci. 2014, 49, 1720-1733.

(55) Brotchie, A.; Grieser, F.; Ashokkumar, M. Effect of Power and Frequency on Bubble-Size Distributions in Acoustic Cavitation. Phys. Rev. Lett. 2009, 102, 084302. 